\title{
Malignant Pigmented Villonodular Synovitis in the Knee - Report of a Case with Rapid Clinical Progression
}

\author{
Naoaki Imakiire ${ }^{1}$, Takashi Fujino ${ }^{2}$, Takeshi Morii ${ }^{*}{ }^{1}$, Keita Honya $^{3}$, Kazuo Mochizuki ${ }^{1}$, \\ Kazuhiko Satomi ${ }^{1}$ and Yasunori Fujioka ${ }^{2}$
}

\author{
Department of ${ }^{1}$ Orthopaedic Surgery, ${ }^{2}$ Pathology, and ${ }^{3}$ Radiology. Kyorin University Faculty of Medicine. 6-20-2 \\ Shinkawa Mitaka Tokyo 181-8611 Japan
}

\begin{abstract}
Malignant pigmented villonodular synovitis (PVNS) (or malignant giant cell tumor of tendon sheath (GCTTS) is an extremely rare condition defined as a malignant lesion occurring with concomitant or previously documented PVNS at the same site. To date, only less than 20 cases have been reported in English literatures. We report a case of malignant PVNS in the knee in a 56-year-old woman with unpredictable rapid progression. This case raised a caution that when atypical components in specimens of recurrent benign PVNS are detected, even if low-grade or tiny, both pathologists and surgeons should consider the risk of malignant PVNS, which could display aggressive clinical progression.
\end{abstract}

Keywords: Soft tissue tumor, malignant pigmented villonodular synovitis, malignant giant cell tumor of tendon sheath.

\section{CASE PRESENTATION}

A 56-year-old woman with a painful mass in the posterior part of the left knee was admitted to a nearby clinic in 1995. Magnetic resonance imaging (MRI) demonstrated a lobular mass (Fig. 1a). Subtotal removal was performed in June 1996 in that clinic under the diagnosis of conventional pigmented villonodular synovitis. Although local recurrence was detected 6 months after surgery, no plan was made for excision because the disease remained stable during the next 10 years. She was followed by the clinic. Later, for diagnosis confirmation, we accessed the specimen from the first operation, revealing marked proliferation of synovia, nodular growth, scattered hemosiderin-laden macrophages and osteoclastic giant cells. No focal sarcomatous components were identified anywhere in the specimen. These findings met the diagnostic criteria for benign PVNS (Fig. 1b, c).

Due to the progressive intolerable pain lasting for 6 months in 2006, she visited our institute. Plain radiography revealed osteolytic lesions in both the femur and the tibia. MRI demonstrated abnormal masses around the knee joint with bone invasion (Fig. 2a). A second excision was therefore performed in December 2006 under a diagnosis of residual PVNS. For extra-articular lesion, marginal resection was performed. Curettage and artificial bone graft were performed for intraosseous lesion. A large part of the specimen from the second operation revealed typical PVNS. However, a subtle nodular myxomatous focus with transition from ordinary PVNS was apparent (Fig. 2b). Atypical spindle cells were seen proliferating in the myxoid stroma (Fig. 2c). Solid nodule of pleomorphic and spindle cells in other aspects with transition from conventional PVNS area

*Address correspondence to this author at the Department of Orthopaedic Surgery, Kyorin University Faculty of Medicine. 6-20-2 Shikawa Mitaka Tokyo 181-8611 Japan; Tel: +81-422-47-5511; Fax: +81-422-48-4206;

E-mails: t-morii@gb3.so-net.ne.jp,t-morii@ks.kyorin-u.ac.jp were confirmed (Fig. 2d, e). In these areas, MIB-1 index (the percentage of Ki-67-positive cells based on a count of 1000 tumor cells within the tumor) was, however, less than $15 \%$. Pleomorphism was not evident. No necrotic lesion was seen throughout the specimen. Thus at that time, it was difficult to determine whether this condition was benign or low grade malignancy and we decided to perform intensive observation at the outpatients' clinic.

During the next 3 months, rapid and aggressive relapse progressed with pathological fractures occurring in the distal femur and proximal tibia (Fig. 3a). Soft tissue invasion was detected up to the proximal part of the thigh (Fig. 3b). Therefore we decided to undergo disarticulation for local control (Fig. 3c, d). The specimen from the third operation showed a destructive multi-nodular growth pattern with myxomatous and solid components. The lesion displayed atypical spindle cells, similar to those in the tiny atypical focus of the second specimen (Fig. 3e). Although a very subtle trace of a benign PVNS-like component was detected, most of the specimen was occupied by low-grade malignant component. High grade component was not detected throughout the specimen. Immunohistochemical studies including S-100, desmin, smooth muscle actin, and CD99, revealed no significant differentiation markers in these components from both second and third operations. Again, MIB-1 index was less than $15 \%$.

Suspected multiple metastases were identified on the first lumbar vertebra and pelvis 11 months after the third surgery. We prescribed a course of postoperative chemotherapy using ifosfamide $\left(10 \mathrm{~g} / \mathrm{m}^{2}\right)$ and doxorubicin $\left(60 \mathrm{mg} / \mathrm{m}^{2}\right)$, following administration of zoledronate and carbon ion radiotherapy for local control of bone metastases. The patient remains alive with the disease 21 months after the third operation without evident lung metastases. The patient was informed that data concerning the case would be submitted for publication, and she consented. 
(a)

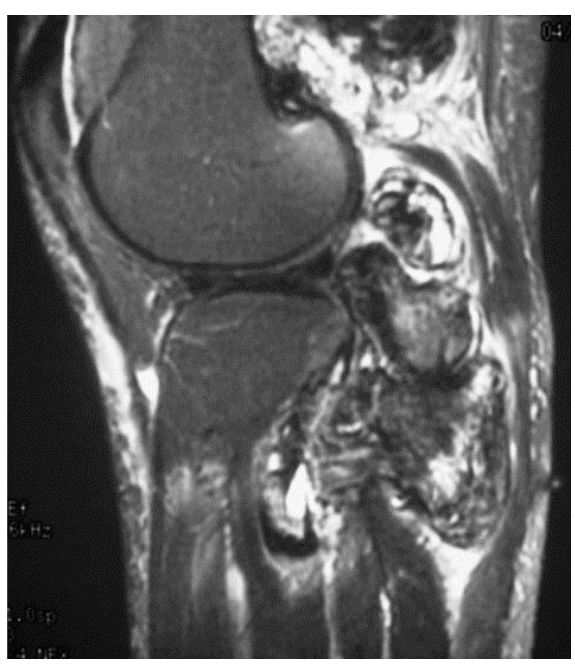

(b)

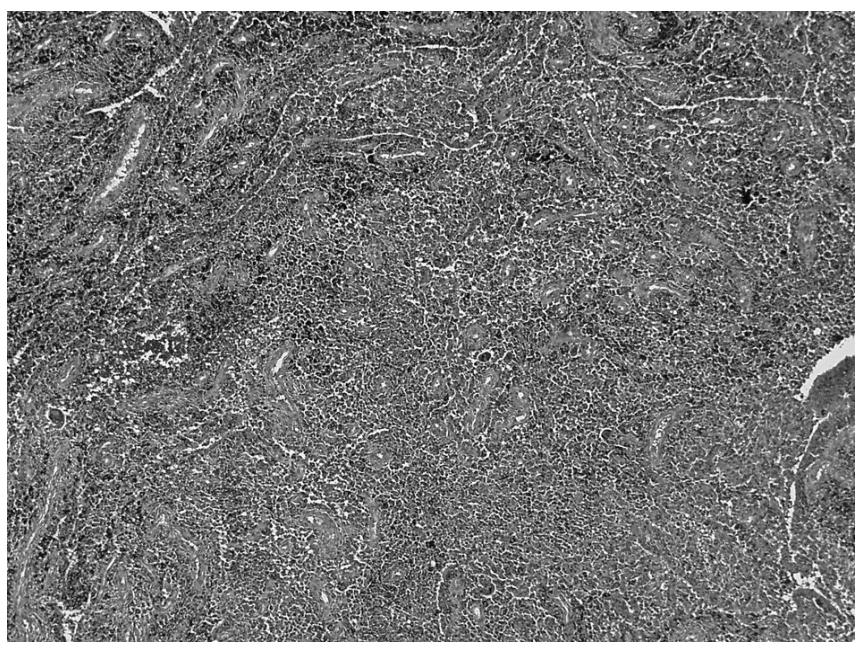

(c)

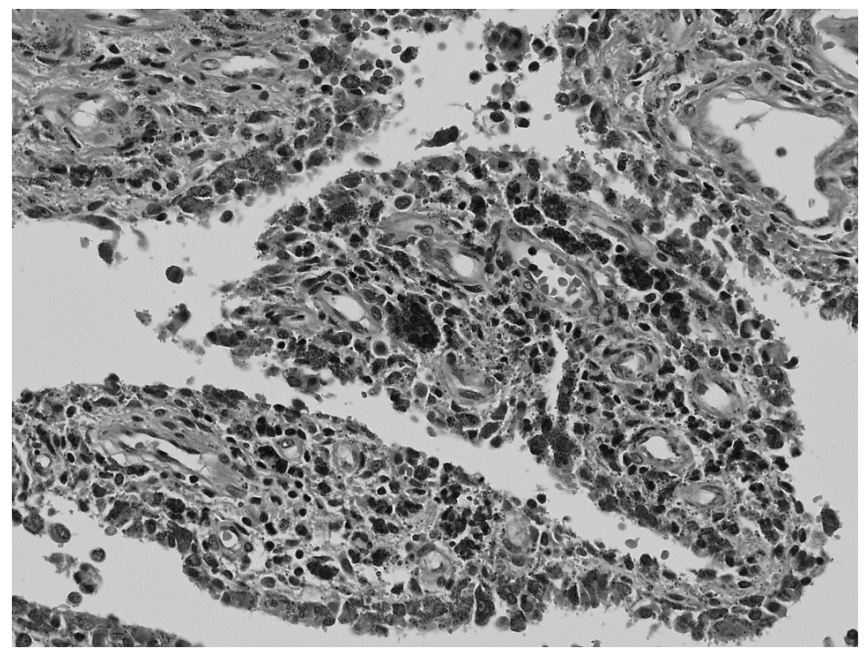

Fig. (1). First operation at nearby clinic. a; MRI before the first excision, showing a well-demarcated lobular mass at the posterior part of the knee. $\mathbf{b}$ and $\mathbf{c}$; Histological findings of the specimen from the first excision, showing conventional PVNS without any trace of malignancy (hematoxylin and eosin stain, b; original magnification $\times 40, \mathrm{c} ; \mathrm{x} 200)$. (a)

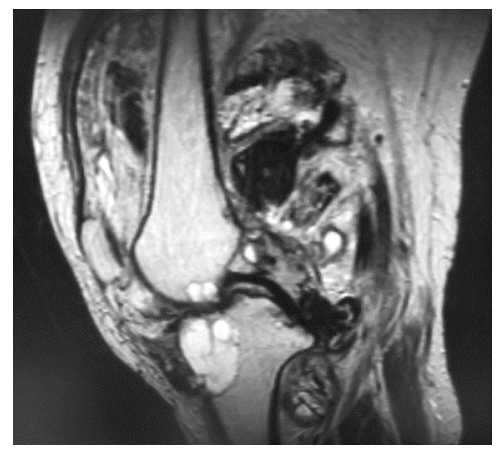

(b)

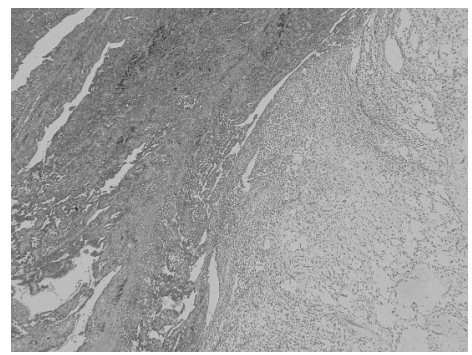

(c)

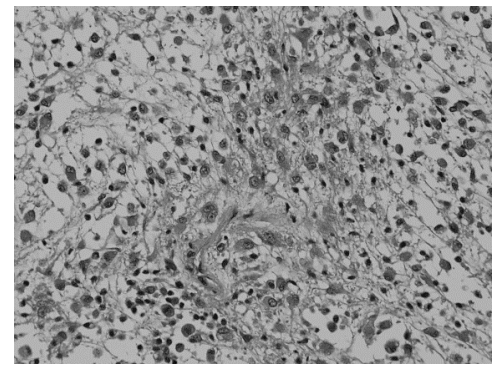

(d)

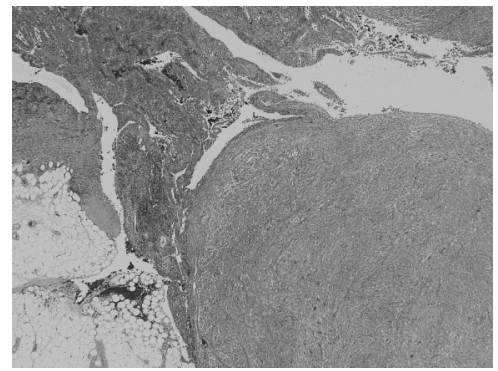

(e)

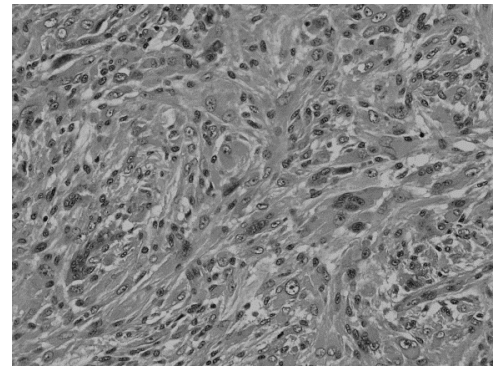

Fig. (2). Second operation. a; MRI showing enlargement of the residual tumor together with bone destruction. b-e: Specimen from the second excision (hematoxylin and eosin stain, b; original magnification x20, c; x200, d; x20, e; x200). 
(a)

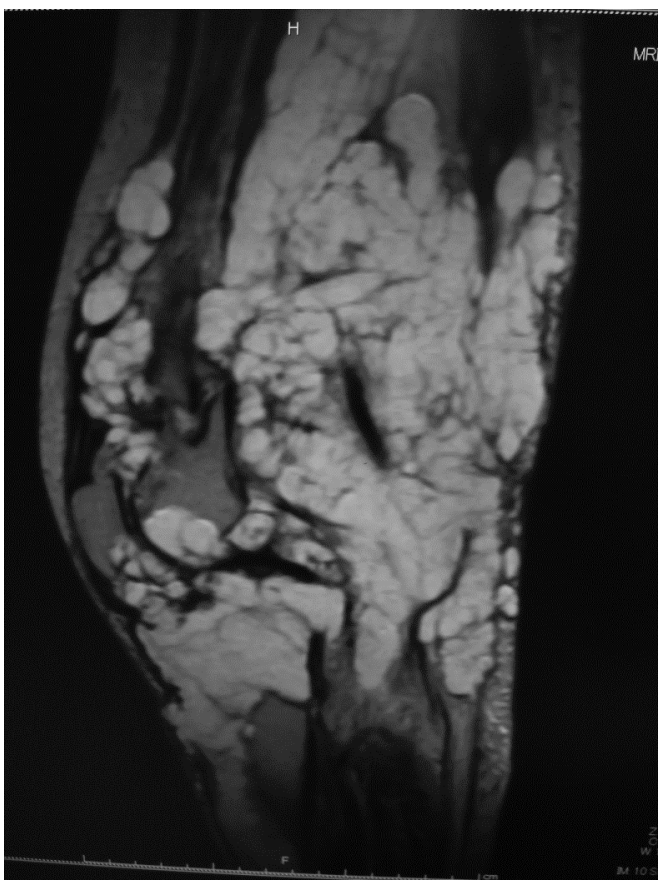

(b)

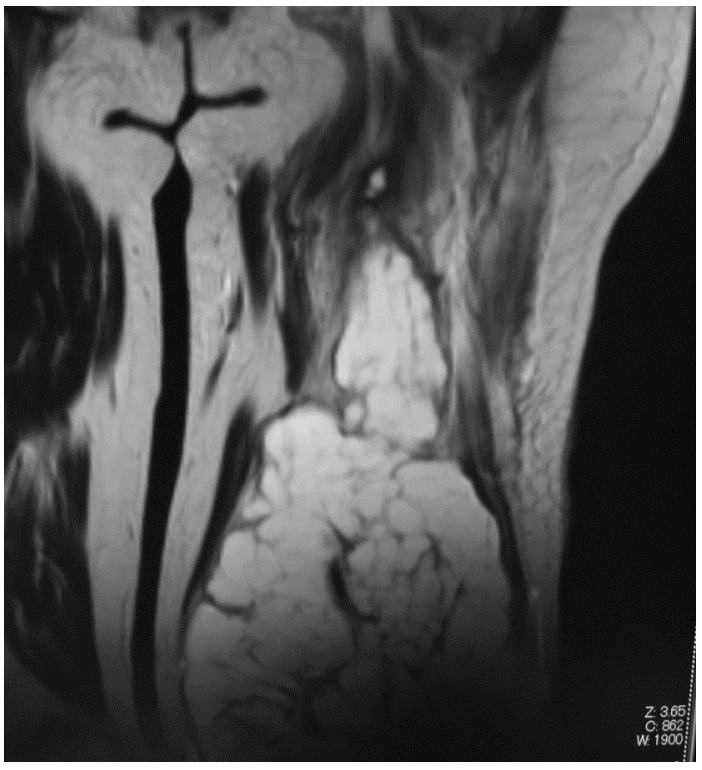

(c)

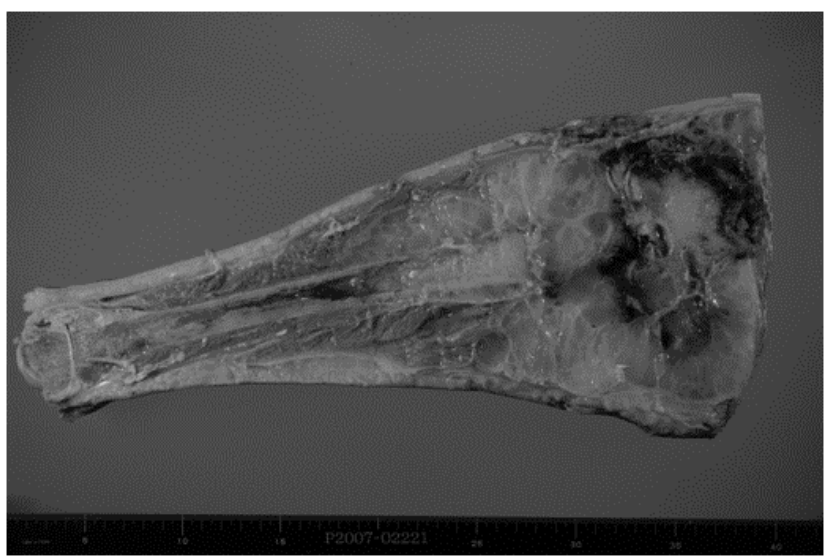

(Fig. 3) contd.....

(d)

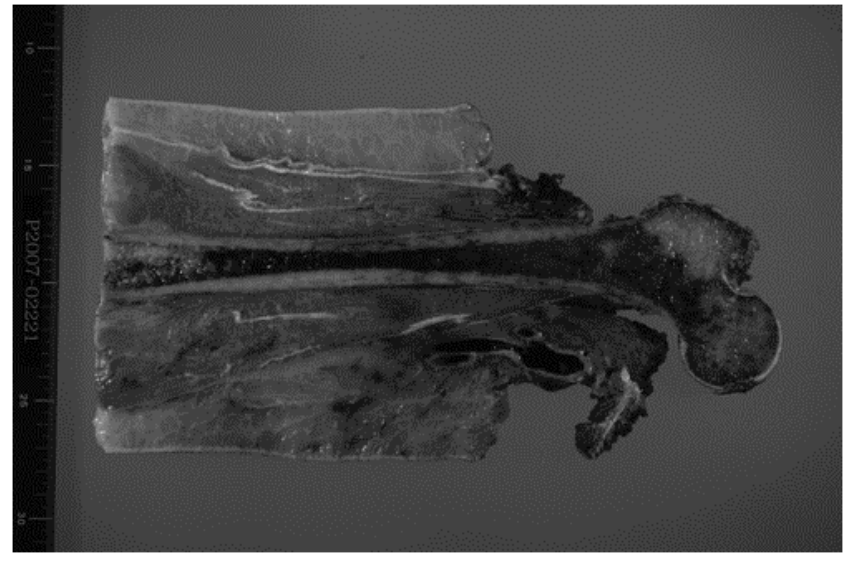

(e)

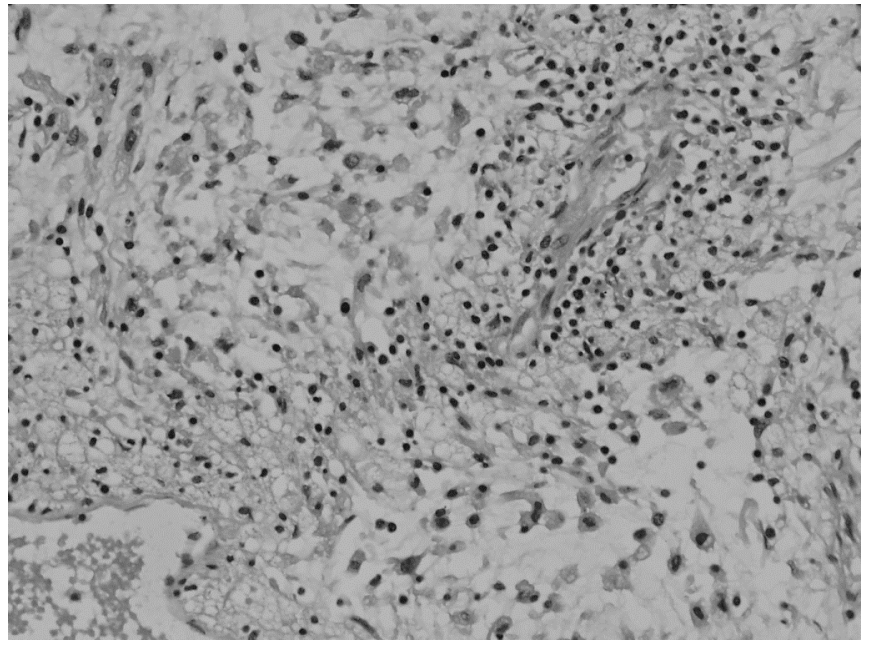

Fig. (3). Third operation. a and $\mathbf{b}$; MRI at 3 months after the second operation. Whole bone occupation is seen, with pathological fracture in the femur. Broad subcutaneous occupation is also apparent (a). Tumor invasion into the proximal part of the thigh (b). c and d; macroscopic findings of the specimen from the disarticulated limb. e; Lobular proliferation of atypical tumor cells, myxoid stroma and dissepiments throughout the specimen (hematoxylin and eosin stain, original magnification x200). No high grade component was detected throughout the specimen.

\section{DISCUSSION}

Malignant PVNS is an extremely rare condition but established concept involving a malignant form of GCTTS or PVNS. Enzinger and Weiss proposed the definition of the condition as a malignant lesion occurring with concomitant or previously documented conventional PVNS at the same site [1]. In the present case, both clinical and histological data met this criterion, warranting the diagnosis of malignant PVNS.

Although some of the previous cases reported as malignant PVNS were not accepted as this entity by ohter authors $[1,2]$, to date, about 20 cases of this entity were reported in English literatures [2-13]. Clinical property of this entity was summarized as follows based on these reports. Their ages ranging from 12 to 78, average 52, years. Most cases involved the knee and the ankle $[2-4,6,8,9]$. 
Almost half of the patients died during the follow up period $[3-6,10]$. Limb salvage was difficult due to repeated local recurrence or joint involvement, and for most cases amputation was indicated [2-6, 8-10]. However in some younger patients' cases, lethal events could be avoided for more than 40 years after the initial treatment in spite of the repeated local recurrence [2-4].

Bertoni et al. analyzed eight typical cases and extracted common histological features: 1) a nodular solid infiltrative growth pattern; 2) large, plump round or oval cells; 3) cells with large nuclei containing prominent nucleoli; 4) necrotic areas; and 5) absence of a zonal pattern of maturation [3]. Although features 1),4) and 5) matched the findings in the present case, some interesting variations were seen in both cell shape and background components, specifically in the form of short spindle cells with nodular myxoid infiltrative pattern. We propose here that the present case represents an example of malignant PVNS with histological features of myxofibrosarcoma. Indeed, cases previously reported as malignant PVNS have displayed some variations in histological findings diverging from the description by Bertoni et al. such as proliferation of spindle-shaped cells [2, 4, 7], myofibroblast-like components [9], osteosarcomatous lesion [11] or myxoid components [4, 14].

The notable feature of the present case was the discrepancy between low-grade histological findings and rapid, extensive clinical progression. Malignant components from both the second and third operation showed a low mitotic rate. Atypia and pleomorphism were not evident. Areas of necrosis were not dominant. All these findings suggested low histological grade. We failed to make rational explanation for the discrepancy, however, examples of aggressive clinical behavior of malignant PVNS with rather low mitotic rate $(<10$ per 10 high-power fields) have been previously reported $[4,10]$. Our experience suggested a strong need for an appropriate and careful pathological work up for discriminating between conventional and malignant PVSN. In cases of less experienced pathologic centers, a transfer to an expert might be kept in mind.

\section{CONCLUSION}

An unusual form of malignant PVNS with myxofibrosarcoma-like components was reported. This case indicated that caution is warranted regarding the probability of unpredictable rapid clinical progression in aggressive or malignant PVNS, even when histological grade appears low.

No competing interests are disclosed.

\section{ACKNOWLEDGEMENT}

We would like to thank Kaoruko Kojima for her expert technical assistance.

\section{REFERENCES}

[1] Enzinger FM, Weiss SW. In: Enzinger FM, Weiss SW Ed, Soft tissue tumors. 3rd ed. Philadelphia, CV Mosby 1995; 749-51.

[2] Layfield LJ, Meloni Ehrig A, Liu K, Shepard R, Harrelson JM. Malignant giant cell tumor of synovium (malignant pigmented villonodular synovitis). Arch Pathol Lab Med 2000; 124(11): 1636-41.

[3] Bertoni F, Unni KK, Beabout JW, Sim FH. Malignant giant cell tumor of the tendon sheaths and joints (malignant pigmented villonodular synovitis). Am J Surg Pathol 1997; 21(2): 153-63.

[4] Kalil RK, Unni KK. Malignancy in pigmented villonodular synovitis. Skeletal Radiol 1998; 27(7): 392-5.

[5] Huracek J, Troeger H, Menghiardi B, et al. Malignant course of pigmented villonodular synovitis of the flexor tendon sheath of the small finger--case report and review of the literature. Handchir Mikrochir Plast Chir 2000; 32(4): 283-90.

[6] Nielsen AL, Kiaer T. Malignant giant cell tumor of synovium and locally destructive pigmented villonodular synovitis: ultrastructural and immunohistochemical study and review of the literature. Hum Pathol 1989; 20(8): 765-71.

[7] Oda Y, Takahira T, Yokoyama R, Tsuneyoshi M. Diffuse-type giant cell tumor/pigmented villonodular synovitis arising in the sacrum: malignant form. Pathol Int 2007; 57(9): 627-31.

[8] Castens HP, Howell RS. Maglignant giant cell tumor of tendon sheath. Virchows Arch A Pathol Anat Histol 1979; 382(2): 237-43.

[9] Ushijima M, Hashimoto H, Tsuneyoshi M, et al. Malignant giant cell tumor of tendon sheath. Report of a case. Acta Pathol Jpn 1985; 35(3): 699-709.

[10] Shinjo K, Miyake N, Takahashi Y. Malignant giant cell tumor of the tendon sheath: an autopsy report and review of the literature. Jpn J Clin Oncol 1993; 23(5): 317-24.

[11] Wu NL, Hsiao PF, Chen BF, Chen HC, Su HY. Malignant giant cell tumor of the tendon sheath. Int J Dermatol 2004; 43(1): 54-7.

[12] Kahn LB. Malignant giant cell tumor of the tendon sheath. Ultrastructural study and review of the literature. Arch Pathol 1973; 95(3): 203-8.

[13] Bliss BO, Reed RJ. Large cell sarcomas of tendon sheath. Malignant giant cell tumors of tendon sheath. Am J Clin Pathol 1968; 49(6): 776-81.

[14] Somerhausen NS, Fletcher CD. Diffuse-type giant cell tumor: clinicopathologic and immunohistochemical analysis of 50 cases with extraarticular disease. Am J Surg Pathol 2000; 24(4): 479-92. 